\title{
Symptome managen in der Onkologie
}

\author{
Von der Theorie in die Praxis - und wieder zurück Das Assessment von \\ krankheits- und therapiebedingten Symptomen sowie individuell angepasste \\ Beratungs- und Symptommanagement-Interventionen sind Kernkompetenzen \\ onkologischer Pflege. Doch Symptome sind subjektive Erfahrungen und für \\ Pflegende nicht immer einfach zu erfassen. Eine Exkursion in die Symptom- \\ theorie kann dabei helfen, Symptome besser zu verstehen und effektive Maß- \\ nahmen abzuleiten. " Matthias Hellberg-Naegele
}

\section{D zUSAMMENFASSUNG}

\begin{abstract}
Ausgangspunkt einer erfolgreichen SymptommanagementIntervention ist die Symptomerfahrung. Diese setzt sich aus verschiedenen Dimensionen zusammen: In welcher Ausprägung zeigt sich ein Symptom, zu welcher Belastung führt es bei den Betroffenen und welche Bedeutung hat es für diese? Mit diesem Verständnis können individuelle Interventionen abgeleitet werden. Symptome können sich aber in verschiedenen Settings unterscheiden, abhängig von den vor Ort verwendeten Symptommanagement-Standards. Ein Praxismodell soll hier zeigen, wie Symptome der Patienten im eigenen Setting identifiziert, die Entstehung verstanden, geeignete Interventionen entwickelt und die Symptomerfahrung verbessert werden kann.
\end{abstract}

\section{Schlüsselwörter: Symptombelastung, Intervention, Symptomerfahrung, Mukositis, Diarrhö}

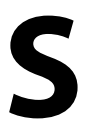
ymptommanagement ist mehr als die Anwendung standardisierter Interventionen. Symptome müssen genau verstanden werden, damit individuelle Lösungen gefunden werden können. Diese müssen dann von Patienten samt Angehörigen im Alltag selbstständig durchgeführt werden. Information, Schulung und Beratung zum Symptomselbstmanagement ist notwendig, vor allem bei Krebserkrankungen, die immer öfter einen chronischen Verlauf haben.

Das Verstehen der Ursachen und der Auswirkungen, welche die Symptome der Patienten in deren Alltag verursachen, muss das Ziel des pflegerischen Assessments sein. Daraus können den Patienten individuell passende Interventionen angeboten werden, die deren Bedürfnissen entsprechen und die Einschränkungen im Alltag beheben, reduzieren oder eine weitere Verschlechterung verhindern. Den Weg zu einer individuell angepassten Intervention beschreibt das Symptommanagement-Modell von Dodd et al. (2001). Darin bildet die Symptomerfahrung der Patienten den Ausgangspunkt.

\section{Was ist ein Symptom?}

Ein Symptom ist eine subjektive Erfahrung, die Veränderungen in der bio-psycho-sozialen Funktion, den Empfindungen oder der Wahrnehmung eines Individuums widerspiegelt (Dodd et al. 2001). Symptome sind also subjektive Phänomene, die den Betroffenen eine Veränderung zum Normalzustand anzeigen. Manche Symptome können zu Beginn ignoriert werden. Manche muss man sich bewusstmachen. Symptome müssen begriffen und verstanden werden, was sie sowohl für das betroffene Individuum als auch für das medizinische Behandlungsteam sehr komplex macht (Rhodes \& Watson 1987). Ein Symptom kann schon eine ganze Weile bestehen, bevor die ersten Anzeichen von Fachpersonen zu erkennen sind. Symptome, z.B. eine orale Mukositis nach einer HochdosisChemotherapie können vorübergehend auftreten. Sie können auch chronisch manifestiert sein, z.B. die Fatigue, die noch Jahre nach einer erfolgreichen Krebsbehandlung vorhanden sein kann. Oder sie sind wiederkehrend wie die antizipatorische Übelkeit nach Chemotherapie.

Letztlich entwickeln sich viele Phänomene von einem subjektiven Symptom hin zu einem sichtbaren Zeichen. Die Chemotherapie-induzierte Polyneuropathie (CIPN) beginnt zum Beispiel rein subjektiv. Betroffene spüren möglicherweise ein Kribbeln in Händen oder Füßen. In diesem Frühstadium ist die CIPN nur erkennbar, wenn Patienten diese äußern. Beobachtbar wird die CIPN erst durch sichtbare Schmerzreaktionen bei der Berührung von Gegenständen. Für wirksame Interventionen ist es in der Regel dann aber 
zu spät. Somit ist die Beratung zur Selbstbeobachtung auf CIPN die wichtigste Intervention - Prävention also durch Symptomberatung. Patienten müssen eine CIPN erkennen und diese ihrem Behandlungsteam frühzeitig melden.

Deutlich sichtbare Anzeichen von Symptomen erhalten in der Patientenbetreuung meist mehr Aufmerksamkeit, weil sie beobachtbar oder sogar messbar sind. Solange sie rein subjektiv sind, sind sie deutlich schwerer zu erkennen und sehr individuell. Die Art und Weise, wie die Symptome erfahren werden, kann von Individuum zu Individuum daher sehr unterschiedlich sein. Deren Symptomerfahrung kann sich von der im Assessment erhobenen Häufigkeit, Intensität oder Dauer unterscheiden. So können im Assessment Symptome erfasst werden, die die Patienten aber nicht beunruhigen wie die Appetitlosigkeit während einer HochdosisChemotherapie (Naegele et al. 2018). Dass diese auftreten kann, ist vielen Patienten schon vor der Therapie bewusst. Die Appetitlosigkeit ist erwartbar und zeitlich begrenzt. Patienten können aber auch von Symptomen beunruhigt sein, die durch das medizinische Team überhaupt nicht festgestellt wurden (McDaniel \& Rhodes 1995). Diese Symptomerfahrung sollte der Ausgangspunkt für das Symptommanagement sein.

\title{
Jedes Symptom hat mehrere Dimensionen
}

Die Symptomerfahrung umfasst mehrere Dimensionen. Eine davon ist die Symptomausprägung. Hierzu zählen deren Häufigkeit, Dauer und Intensität bzw. Ausmaß, indem ein Symptom Beschwerden verursacht (McDaniel \& Rhodes 1995). Diese Faktoren lassen sich durch die Common Terminology Criteria for Adverse Events (CTCAE) des National Cancer Institute darstellen (National Cancer Institute 2017). Hier findet eine Gradeinteilung für zahlreiche Symptome statt, zum Beispiel anhand der Frequenz oder der Intensität der Beeinträchtigung der Aktivitäten des täglichen Lebens (ATL) oder deren Bedrohlichkeit. Grad 1 ist dabei meistens die erste Erscheinung des Symptoms ohne direkten Einfluss auf den Gesundheitsstatus. Grad 2 ist häufig definiert durch Einschränkungen in den instrumentellen Aktivitäten des täglichen Lebens (IATLs) (Lawton \& Brody 1969), Grad 3 durch Einschränkungen in den ATLs (Katz et al. 1963), Grad 4 ist ein lebensbedrohlicher Zustand und Grad 5 ist immer der Tod durch das Symptom. In aller Regel gelten Symptome mit Grad 1 oder 2 als tolerabel.

Doch ob ein Symptom tolerabel ist oder nicht, ist nur anhand der Symptomausprägung nicht beurteilbar. Denn Patienten können ihre Symptome sehr unterschiedlich bewerten. Während für den einen ein Grad 2-Symptom tolerabel ist, kann für den anderen Patienten ein Grad 2-Symptom nicht tolerierbar sein und ihn zum Abbruch einer Therapie bewegen. Der Unterschied liegt in der Belastung, die durch ein Symptom beim Patienten entsteht. Die Symptombelastung ist die Reaktion eines Menschen auf das Ausmaß eines Symptoms. Sowohl die Abweichung von der normalen Funktion, Empfindung oder Erscheinung als auch die Interpretation deren Bedeutung bestimmt die Symptombelastung (McDaniel \& Rhodes 1995). Ein wichtiger Schritt zum Erfassen der Patientenperspektive auf Symptome ist die Verwendung von Patient-Reported Outcomes (PRO). Hier können zur Erfassung der Symptomausprägung die PRO CTCAEs des National Cancer Institutes verwendet werden (National Cancer Institute 2020). Zur Erfassung der Sym-

\section{Hier steht eine Anzeige.}

\author{
Springer
}


Abb. 1: Modell für die Praxis zur Entwicklung von Symptommanagement-Programmen

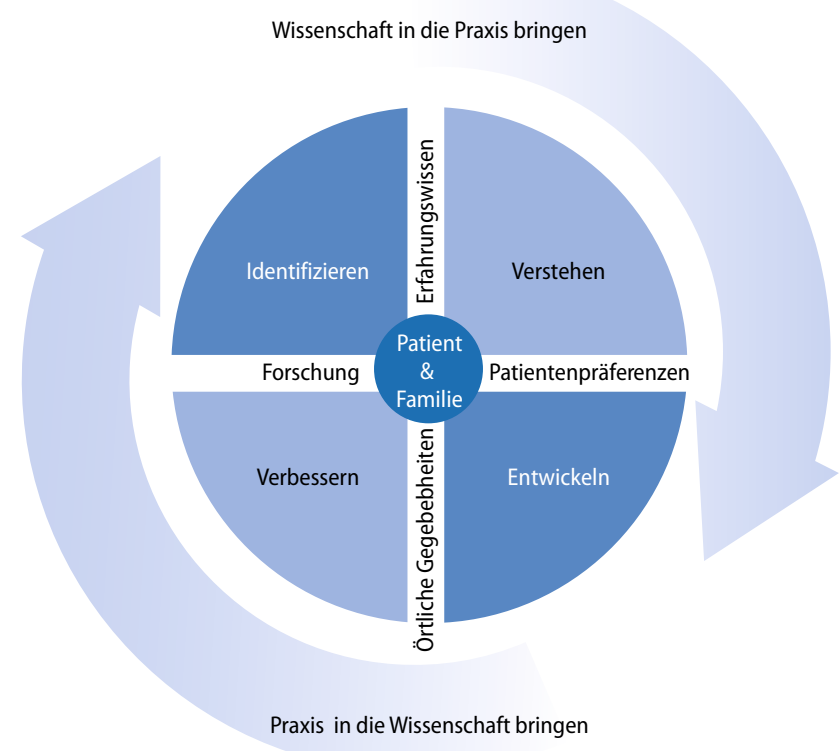

ptombelastung können die Fragen des PRO CTCAE-Kataloges umformuliert werden. Die Frage lautet dann, wie belastend ein Symptom in den letzten sieben Tagen war (Kirsch et al. 2011; Naegele et al. 2018).

Das Konzept der Symptomerfahrung vereinigt die Ausprägung und die Belastung miteinander. So wurde die Symptomerfahrung zunächst definiert als die Wahrnehmung und Reaktion von Patienten auf ihre Symptomausprägung und deren Belastung (Simms et al. 1993). Symptome stellen also die Erkrankung so dar, wie sie von einem Individuum im Rahmen eines kognitiven Bearbeitungsprozesses erfahren wird (Armstrong 2003).

\section{Intensität und Belastung können gegenläufig sein}

Ein intensiv empfundenes Symptom muss aber nicht zwangsläufig auch als belastend wahrgenommen werden. Intensität und Belastung können sogar gegenläufig sein. Ein Patient kann auch aufgrund eines Symptoms, das er nicht hat, belastet sein. Dies kann bei Symptomen auftreten, die mit der Wirkung eines Medikaments korrelieren, beispielsweise bei Diarrhö während der Erhaltungstherapie mit Lenalidomid bei Patienten mit Multiplem Myelom. Das Ausbleiben der Diarrhö könnte durch den Patienten gleichgesetzt werden mit der Unwirksamkeit der Therapie, was ihn sehr belastet. Das entscheidende Kriterium ist hier die Bedeutung, die einem Symptom zugesprochen wird. Die Symptombedeutung ist stark geprägt von Vorerfahrungen oder der Kultur. Sie macht die Symptomerfahrung zu einem multidimensionalen und dynamischen Prozess, dessen Bedeutung ein Individuum von einer subjektiven, ungewöhnlichen Empfindung ableitet.

Im Symptoms Experience Modell definiert Armstrong die Symptomerfahrung als die Wahrnehmung der Häufigkeit, der Intensi- tät, der Belastung und der Bedeutung von Symptomen, die bei deren Entstehung auftritt (Armstrong 2003). Häufig treten sogar mehrere Symptome gleichzeitig auf, die sich in ihrer Wirkung auf die Symptomerfahrung multiplizieren können. Als beeinflussende Faktoren beschreibt sie die demographischen Eigenschaften von Patienten und die Charakteristika ihrer Erkrankung sowie individuelle Eigenschaften. Die Art und Weise, wie sich die Symptomerfahrung ausdrückt, hängt von den Konsequenzen des Symptoms ab, wie der Anpassungsfähigkeit an die Erkrankung, die Lebensqualität, Stimmung, funktioneller Status, Krankheitsprogression oder Überleben. Das Armstrong Modell macht deutlich, wie breit ein individuelles Symptom-Assessment aufgestellt sein muss, um die Symptomerfahrung eines Patienten zu verstehen. Hinzu kommt der Faktor Zeit. Ein Symptom, welches über eine lange Zeit erlebt wird, kann auf Dauer unterschiedlich bewertet werden. Deshalb fügte Armstrong den zeitlichen Verlauf der Symptome als weitere Ebene in ihr Modell ein (Armstrong 2014).

\section{Ein Symptommanagement-Modell für die Pflegewissenschaft}

Im Symptommanagement-Modell von Dodd et al. bildet die Symptomerfahrung der Patienten die Grundlage für das Symptommanagement (Dodd et al. 2001). Es basiert folglich auf Patient Reported Outcomes, eine der Hauptvoraussetzungen in diesem Modell. Bei manchen Symptomen kann aber auch eine präventive Intervention sinnvoll sein, beispielsweise, wenn durch eine Intervention ein Symptom verhindert werden kann wie die hochgradige orale Mukositis durch die orale Kryotherapie bei der Hochdosis Melphalan (Leppla, Naegele, Rebafka et al. 2016).

Symptome müssen jedoch auch bei Personen, die sich nicht äußern können, behandelt werden. Die Grundlage für Interventionen bilden dann die Interpretationen von Eltern oder Fachpflegenden. Eine Symptommanagement-Intervention kann auf ein Individuum, dessen Familie, eine Gruppe oder ein Setting abzielen. Und das Symptommanagement ist ein dynamischer Prozess, der durch individuelle Outcomes und Einflüsse der Dimensionen pflegerischen Handelns (Mensch, Gesundheit und Krankheit, Umwelt) verändert werden kann. Die Dimensionen pflegerischen Handelns stammen aus der Welt der Pflegetheorien, die die Verbindung zur Pflegewissenschaft ermöglichen sollen. Das ist zur Entwicklung von Symptommanagement-Interventionen essenziell. Der Mensch reagiert aufgrund seiner demographischen, psychologischen, soziologischen und physiologischen Variablen individuell auf die Symptome. Der Gesundheits- oder Krankheitsstatus eines Menschen mit allen Einflussfaktoren wie Risikofaktoren, Verletzungen oder Behinderungen beeinflusst die Symptomerfahrung. Und die Umwelt ist die Summe aus allen Umständen, unter denen ein Symptom auftritt. Das umfasst physische (Zuhause, Arbeit, Krankenhaus), soziale (unterstützendes Netzwerk, Beziehungen) und kulturelle (Glaube, Werte, Gepflogenheiten) Umstände.

Im Kontext dieser Dimensionen spielt sich das Symptom-Management ab, ausgehend von der Symptomerfahrung. Ziel ist es, Symptome zu verhindern oder abzuschwächen. Hierfür ist das Erheben der Symptomerfahrung essenziell. Hieraus werden Interventionen abgeleitet, die auf eine oder mehrere Komponenten der individuellen Symptomerfahrung abzielen. Symptommanagement ist 
dabei ein dynamischer Prozess, der immer wieder Änderungen der Interventionsstrategien erfordern kann. Im Modell von Dodd et al. (2001) werden Symptom-Management-Strategien charakterisiert durch: Was wird getan? Wann? Wo? Warum? Wie viel? Für wen? Wie? Der Outcome des Symptom-Managements kann durch Erheben des funktionellen oder emotionalen Status, der Fähigkeit zur Selbstpflege, Kosten, Lebensqualität, Morbidität und Mortalität evaluiert werden.

\section{Symptommanagement-Modell in der Praxis}

Welche Symptome Patienten bei bestimmten Therapien erfahren müssen, kann zwischen verschiedenen Kliniken unterschiedlich sein und ist abhängig von den vor Ort angewandten Symptommanagement-Interventionen. Daher müssen im Rahmen des Symptommanagements nicht nur wissenschaftliche Erkenntnisse in die Praxis gebracht werden, sondern auch praxisrelevante Probleme mit wissenschaftlichen Methoden analysiert werden. Aus diesem Grund gewann die Implementierungs-Forschung, die auf dieser zirkulären Beziehung zwischen Theorie und Praxis aufbaut, in den letzten Jahren immer mehr an Bedeutung. Ein in der Klinik für Hämatologie, Onkologie und Stammzelltransplantation der Uniklinik Freiburg entstandenes Modell (Abb. 1) zeigt in vier Schritten, dass Symptome vor Ort erst identifiziert und dann verstanden werden müssen. Daraufhin kann eine Intervention entwickelt werden, die im letzten Schritt zu einer Verbesserung der Symptomerfahrung der Patienten führt (Leppla, Naegele, Mößner et al. 2016).

Am Beispiel von Patienten mit Multiplem Myelom während der Hochdosischemotherapie Melphalan und autologer Stammzelltransplantation wurde die Symptomerfahrung erfasst und Diarrhö als sehr intensives und sehr belastendes Symptom identifiziert (Naegele et al. 2018). Anhand vergleichbarer Studien ließ sich dies nicht erklären. Es musste also ein lokales Problem als Folge der in diesem Setting verwendeten Symptommanagement-Strategien sein. Um das Auftreten der Diarrhö besser zu verstehen, musste nach den Ursachen gesucht werden. Handelt es sich wirklich um Diarrhö oder wird es nur als solche empfunden? Wenn ja, von welchem Grad der Diarrhö reden wir? Welchen Verlauf nimmt die Diarrhö? Wo kann eine Intervention ansetzen? Handelt es sich um eine gastrointestinale Mukositis? Ist es eine Nebenwirkung der Antibiotika? Welche Rolle spielen pathogene Keime? Mit Hilfe dieser Fragen konnte eine Symptommanagement-Intervention entwickelt werden.

Die Patienten wurden bereits vor der Hochdosistherapie über das Auftreten der Diarrhö aufgeklärt und ihnen wurde nahegelegt, Loperamid beim Betreuungsteam frühzeitig einzufordern. Parallel dazu wurde das Betreuungsteam nochmals durch Schulungen zum Thema Diarrhö sensibilisiert. In der Evaluation anhand der CTCAE-Kriterien konnte eine Verbesserung des Outcomes gezeigt werden, in dem keine Grad 3-Diarrhö mehr auftrat und die Dauer der Diarrhö verkürzt war (Naegele et al. 2017).

\section{FAZIT}

Ausgangspunkt für das Symptommanagement ist die Symptomerfahrung, die sich aus der Symptomausprägung, der Symptombelastung und der Symptombedeutung zusammensetzt.

Das Symptommanagement-Modell nach Dodd et al. bietet der Pflegewissenschaft und der Pflegepraxis eine Hilfestellung zur Entwicklung von Symptommanagement-Interventionen.

Symptome mit ausgeprägter Symptomerfahrung müssen identifiziert und verstanden werden. Dann kann eine Intervention entwickelt und evaluiert werden.

Die Literaturliste finden Sie online auf springerpflege.de und über das eMag der PflegeZeitschrift.

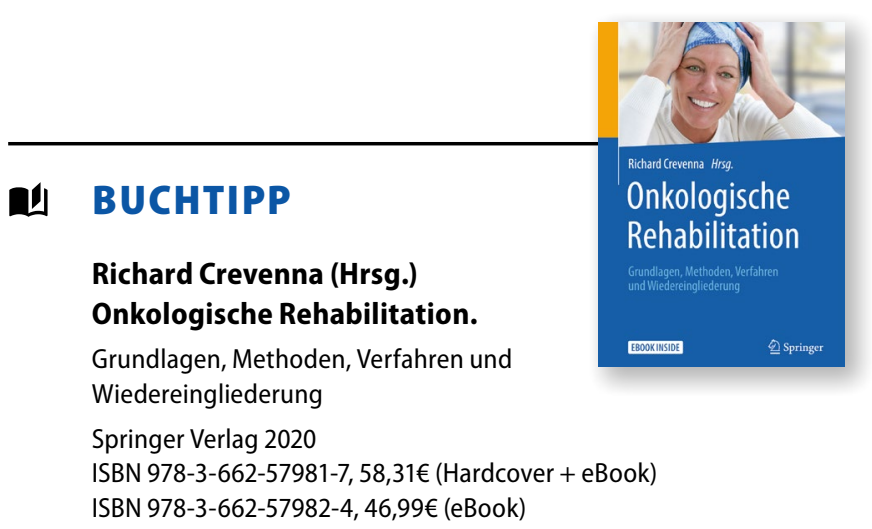

\section{Autorenkontakt:}

Matthias Hellberg-Naegele, M.Sc. Pflegeexperte APN, arbeitet am Comprehensive Cancer Center und in der Klinik für Medizinische Onkologie und Hämatologie am Universitätsspital Zürich matthias.hellberg-naegele@usz.ch 